\title{
Superação de dormência em sementes de Jatobá avaliadas por análise de sobrevivência
}

\author{
Dormancy overcoming in Jatobá seeds assessed by survival analysis
}

\section{Cristiano Nunes Nesi ${ }^{1,2^{*}}$, Guilherme Oliveira Santos Ferraz de Arruda ${ }^{2}$ e Alexandre Menegatti ${ }^{2}$}

Recebido em 21/08/2015 / Aceito em 29/02/2016.

\section{RESUMO}

Este trabalho avaliou métodos de superação de dormência de sementes de Jatobá (Hymenaea courbaril L.) utilizando-se análise de sobrevivência. Avaliaram-se 75 sementes por tratamento: testemunha (T1); imersão em água à temperatura ambiente durante 120 h (T2) e 72 h (T3); imersão em água a $90{ }^{\circ} \mathrm{C}$ e repouso nesta por $24 \mathrm{~h}$ (T4); escarificação mecânica (T5); escarificação mecânica e imersão em água por $24 \mathrm{~h}$ (T6). Diariamente foram anotadas as sementes germinadas por tratamento. Empregou-se o método de Kaplan-Meier para estimar curvas de sobrevivência e tempos medianos para germinação, o teste log-rank e o modelo semiparamétrico de Cox para estimar o efeito dos tratamentos. Os tempos medianos de germinação foram 56 dias (T4) e 33 dias (T6). Sementes submetidas a T4, T5 e T6 têm, respectivamente, 22,5, 4,7 e 3,7 vezes maior capacidade de germinar (tempo para germinação mais curto) que sementes não submetidas à superação de dormência.

PALAVRAS-CHAVE: Hymenaea courbaril, escarificação, tempo-ocorrência, Kaplan-Meier, regressão de Cox.

\section{ABSTRACT}

This work evaluated methods of overcoming dormancy in Jatoba seeds (Hymenaea courbaril L.) by utilizing survival analysis. 75 seeds per treatment were evaluated: without treatment (T1), immersion in water at outdoor temperature for $120 \mathrm{~h}$ (T2) and $72 \mathrm{~h}$ (T3), immersion in water at $90^{\circ} \mathrm{C}$ and rest in the same water for $24 \mathrm{~h}$ (T4), mechanical scarification (T5) and mechanical scarification and soaking it in water for $24 \mathrm{~h}$ (T6). The seeds germinated per treatment were registered daily. The Kaplan-Meier method was utilized to estimate the curves of survival and median periods for the germination, the log-rank test and the Cox semiparametric model to estimate the effect of the treatments. The median periods of germination were 56 days (T4) and 33 days (T6). The seeds submitted to T4, T5 and T6 have respectively $22.5,4.7$ and 3.7 times higher capacity to germinate (shorter period for germination) than those seeds that were not submitted to overcoming dormancy.

KEYWORDS: Hymenaea courbaril, scarification, time-occurrence, Kaplan-Meier, Cox regression.

\section{INTRODUÇÃO}

A dormência é um impedimento para a germinação das sementes, benéfica às espécies devido à distribuição da germinação ao longo do tempo, aumentando a probabilidade da espécie sobreviver (FOWLER \& BIANCHETTI 2000), pois possibilita que a semente germine apenas no momento em que as condições para o estabelecimento das plântulas estejam adequadas (FINCH-SAVAGE \& LEUBNERMETZGER 2006). Embora seja uma estratégia de sobrevivência da espécie, a dormência às vezes é tida como uma característica indesejável para os produtores de mudas florestais, pois pode dificultar ou inviabilizar a germinação (LEMOS FILHO et al. 1997), necessitando ser superada para se obter emergência uniforme (ANDRADE et al. 2010). A impermeabilidade do tegumento, a condição do embrião (imaturo, rudimentar ou dormente), a presença de substâncias inibidoras ou a combinação destes fatores são as causas principais da dormência em sementes florestais. Nas sementes de Jatobá

\footnotetext{
${ }^{1}$ Empresa de Pesquisa Agropecuária e Extensão Rural de Santa Catarina, Chapecó, SC, Brasil.

${ }^{2}$ Universidade do Oeste de Santa Catarina, Xanxerê, SC, Brasil.

*Autor para correspondência <cristiano@epagri.sc.gov.br>.
} 
(Hymenaea courbaril), como em muitas fabáceas, a dormência é causada por um bloqueio físico representado pelo tegumento resistente e impermeável, o qual impede a entrada de água e trocas gasosas, não permitindo embebição da semente nem oxigenação do embrião (ALMEIDA et al. 2011). No entanto, esta dormência pode ser superada de forma natural ao longo do tempo ou acelerada mediante processos químicos, mecânicos, térmicos e hidratação.

Dentre os principais métodos para superar dormência em sementes estão a escarificação química (ácido sulfúrico, ácido clorídrico, hidróxido de sódio); escarificação mecânica (abrasão sobre superfícies ásperas); estratificação (tratamento úmido à baixa temperatura); choque de temperatura (alternância de temperaturas com grande amplitude) e a imersão em água quente $\left(90^{\circ} \mathrm{C}\right)$ por tempos variáveis conforme a espécie (BUSATTO et al. 2013).

Em estudos de superação de dormência, técnicas de análise estatística convencional podem não ser apropriadas quando se avalia o tempo para germinação de sementes, pois o tempo de observação pode terminar antes que todas as sementes sob estudo germinem (censura à direita), observando-se parcialmente a resposta. Além disso, o tempo para ocorrência de um evento frequentemente não segue distribuição normal. Geralmente, dados desta natureza são submetidos à análise estatística convencional, o que limita a capacidade de inferência. A análise de sobrevivência utiliza o método da verossimilhança para estimação de parâmetros e efetivamente extrair informações relevantes e estimativas confiáveis, mesmo em situações com censura, pois descartar observações censuradas reduz o poder dos testes estatísticos devido à perda de graus de liberdade e introduz viés nas funções de sobrevivência (COLOSIMO \& GIOLO 2006), além de superestimar o risco, pois o tempo até a ocorrência do evento é desconhecido (CARVALHO et al. 2011).

Diante disso, o objetivo deste trabalho foi avaliar o efeito de métodos de superação de dormência em sementes de $H$. courbaril utilizando-se a técnica estatística de análise de sobrevivência.

\section{MATERIAL E MÉTODOS}

O estudo foi realizado em casa de vegetação não climatizada entre abril e setembro de 2012, em clima Subtropical (mesotérmico úmido com verão quente) tipo Cfa (Koeppen) com temperatura média anual $16-17{ }^{\circ} \mathrm{C}$ e inserido no bioma Mata Atlântica. Testaram-se seis tratamentos para superação de dormência, cada um com 75 sementes de Jatobá (H. courbaril), onde cada semente representou uma repetição. As sementes foram extraídas de frutos maduros coletados em Holambra-SP e armazenadas de acordo com informações de CARVALHO et al. (2006). Foram selecionadas sementes visualmente uniformes e aparentemente sadias, colocadas em solução de hipoclorito de sódio a 5\% por 30 minutos para desinfestação, sendo deixadas para secar à sombra sobre papel toalha. Os métodos para superação de dormência definidos para este estudo (Tabela 1) foram aplicados seguindo um cronograma, de modo que todos os tratamentos fossem instalados no mesmo dia.

Após a superação da dormência, realizou-se a semeadura em tubetes cônicos de polipropileno de $290 \mathrm{~cm}^{3}$ utilizando-se como substrato vermiculita de granulometria média, com uma semente por recipiente. $\mathrm{O}$ delineamento experimental foi inteiramente casualizado e os tubetes, identificados com os tratamentos e repetição, foram colocados em mesas metálicas móveis com tela de arame galvanizado. Fezse a irrigação automatizada seis vezes ao dia durante dois minutos ininterruptos, utilizando-se aspersores a 1,20 $\mathrm{m}$ do solo.

Todas as sementes foram avaliadas diariamente durante 150 dias, anotando-se as datas da primeira visualização da emissão do caulículo e da abertura da primeira folha definitiva, para obter o período de tempo decorrido desde a semeadura. Terminada a coleta de dados, calculou-se o percentual de germinação em cada tratamento e submeteram-se os dados à técnica de análise estatística denominada análise de sobrevivência.

O método de Kaplan-Meier foi empregado para estimar as curvas de sobrevivência e os tempos medianos para germinação. A função de sobrevivência (Equação 1) é estimada empiricamente por:

$$
\hat{S}(t)=\prod_{i=1}^{n}\left(1-\frac{d_{i}}{n_{i}}\right)
$$

em que $\mathrm{d}_{\mathrm{i}}$ é o número de sementes germinadas em um dado tempo e $n_{\mathrm{i}}$ é o número de sementes no tratamento (CARVALHO et al. 2011).

Com o objetivo de comparar os tratamentos, aplicou-se o teste log-rank, que utiliza a estatística qui-quadrado para comparar os valores observados e esperados de cada tratamento sob a hipótese de que 
Tabela 1 - Tratamentos em sementes de Jatobá (Hymenaea courbaril L.) para superação de dormência em casa de vegetação.

Table 1 - Treatments in Jatoba seeds (Hymenaea courbaril L.) for overcoming dormancy in greenhouse.

\begin{tabular}{|c|c|}
\hline TRATAMENTO & DESCRIÇÃO \\
\hline T1 & Sementes não imersas em água e não escarificadas (Testemunha); \\
\hline $\mathrm{T} 2$ & Sementes imersas em água à temperatura ambiente por $120 \mathrm{~h}$; \\
\hline $\mathrm{T} 3$ & Sementes imersas em água à temperatura ambiente por $72 \mathrm{~h}$; \\
\hline $\mathrm{T} 4$ & $\begin{array}{l}\text { Sementes imersas em água à temperatura de } 90{ }^{\circ} \mathrm{C} \text { e deixadas em repouso } \\
\text { na mesma água por } 24 \mathrm{~h} \text {; }\end{array}$ \\
\hline T5 & Sementes escarificadas em $2 \mathrm{~mm}$ numa extremidade, com grosa 8"; \\
\hline T6 & $\begin{array}{l}\text { Sementes escarificadas em } 2 \mathrm{~mm} \text { numa extremidade com grosa } 8 \text { " e } \\
\text { imersas em água à temperatura ambiente por } 24 \mathrm{~h} \text {. }\end{array}$ \\
\hline
\end{tabular}

a capacidade de germinação é a mesma em todos os tratamentos. Isto equivale a testar se o tempo para germinação das sementes é semelhante entre os tratamentos. Partindo desse pressuposto chamado de riscos proporcionais, estimou-se o efeito dos tratamentos, sem fazer suposições sobre a distribuição de probabilidades do tempo para germinação, utilizando-se o modelo semiparamétrico de Cox (Equação 2) dado por:

$$
\lambda_{j}(t)=\lambda_{0 j}(t) e^{x \beta}
$$

em que $\lambda_{\mathrm{j}}(\mathrm{t})$ é a função de risco, $\lambda_{0 \mathrm{j}}(\mathrm{t})$ é o risco basal, $\mathrm{x}$ é o vetor de covariáveis (tratamentos) e $\beta$ é o vetor de parâmetros a serem estimados.

O modelo de Cox é flexível porque não requer que se assuma uma distribuição de probabilidades particular para a função de risco basal $\left(\lambda_{0 j}(t)\right)$ e assume apenas que as covariáveis agem multiplicativamente sobre o risco, sendo esta a parte paramétrica do modelo (CARVALHO et al. 2011). Para o ajuste do modelo de Cox, o tratamento testemunha (T1) foi fixado como referência para as comparações. As análises foram realizadas com o sistema estatístico $\mathrm{R}$ (R DEVELOPMENT CORE TEAM 2014), utilizando-se os procedimentos do pacote survival (THERNEAU 2014).

\section{RESULTADOS E DISCUSSÃO}

A escarificação mecânica seguida de imersão em água à temperatura ambiente por 24 h (T6), mostrou-se o tratamento mais eficiente na germinação das sementes, com 85,33\% de germinação completa, além das 5,34\% que iniciaram a germinação mas morreram antes de emitir folhas. Sementes que iniciaram a germinação e morreram por fatores desconhecidos atingiram no máximo $5 \%$, sem relação com os tratamentos (Tabela 2). Em pesquisa de germinação de sementes de $H$. courbaril sem superação de dormência, CARVALHO FILHO et al. (2003) obtiveram 47\% de germinação sob pleno sol e $26 \%$ sob tela sombreadora ( $50 \%$ de sombra) aos 180 dias após semeadura, o que evidencia a necessidade de tratamento para superação de dormência. FOWLWER \& BIANCHETTI (2000), reforçam a necessidade de tratamentos das sementes dormentes para que os embriões encontrem condições ambientais favoráveis e retomem seu crescimento, acelerando a germinação.

Os tempos medianos para germinação foram estimados em 56 e 33 dias para T4 e T6, respectivamente, e não foi possível estimá-los para os demais tratamentos, pois mais de $50 \%$ das sementes não germinaram dentro do período de observação (Tabela 3). No presente estudo, os percentuais de germinação das sementes (Tabela 2) tratadas com imersão em água à temperatura ambiente foram inferiores aos obtidos por CARVALHO FILHO et al. (2003), mesmo com períodos 5 vezes e 3 vezes maiores de imersão (120 h e 72 h). Entretanto, a variação nos resultados de germinação para uma mesma espécie pode ser atribuída a fatores ambientais, genéticos e fisiológicos que influenciam na formação da semente (ALEXANDRE et al. 2009).

A comparação entre os tratamentos pelo teste log-rank apresentou a estatística $\chi^{2}$ significativa para o tempo de germinação, rejeitando-se a hipótese da nulidade e concluindo que pelo menos um tratamento 
Tabela 2 - Percentuais de germinação das sementes de Jatobá ao final de 150 dias após a semeadura em função dos tratamentos para superação de dormência.

Table 2 - Germination percentage of Jatoba seeds in the end of 150 days after sowing depending on treatments for overcoming dormancy.

\begin{tabular}{|c|c|c|c|c|}
\hline Tratamento & G.C. & I.G.M. & S.I.G. & N.I.G. \\
\hline (T1) Testemunha & 12,00 & 4,00 & 1,33 & 82,67 \\
\hline (T2) Imersão em água por $120 \mathrm{~h}$ & 16,00 & 0,00 & 2,67 & 81,33 \\
\hline (T3) Imersão em água por $72 \mathrm{~h}$ & 10,67 & 0,00 & 0,00 & 89,33 \\
\hline (T4) Imersão em água a $90^{\circ} \mathrm{C}$ & 56,00 & 2,67 & 1,33 & 40,00 \\
\hline (T5) Escarificação (2 mm) & 46,67 & 0,00 & 0,00 & 53,33 \\
\hline (T6) T5 + imersão em água 24 h & 85,33 & 5,34 & 0,00 & 9,33 \\
\hline
\end{tabular}

G.C: Germinação completa. I.G.M: Iniciou germinação e morreu. S.I.G: Só iniciou germinação (até a última data de observação). N.I.G: Não iniciou germinação.

Tabela 3 - Tratamentos para superação de dormência, número total de sementes e de sementes germinadas, seguidas dos respectivos tempos medianos (em dias) para germinação.

Table 3 - Treatments for overcoming dormancy, total number of seeds and sprouted seeds, followed by the respective median times (in days) for germination.

\begin{tabular}{|c|c|c|c|c|}
\hline \multirow[t]{2}{*}{ Tratamento } & \multirow[t]{2}{*}{ Total } & \multicolumn{2}{|c|}{ Germinadas* } & \multirow[t]{2}{*}{ Tempo } \\
\hline & & Obs. & Esp. & \\
\hline (T1) Testemunha & 75 & 13 & 37,1 & - \\
\hline (T2) Imersão em água por $120 \mathrm{~h}$ & 75 & 14 & 36,3 & - \\
\hline (T3) Imersão em água por $72 \mathrm{~h}$ & 75 & 08 & 37,8 & - \\
\hline (T4) Imersão em água a $90^{\circ} \mathrm{C}$ & 75 & 45 & 29,6 & 56 \\
\hline (T5) Escarificação (2 mm) & 75 & 35 & 29,4 & - \\
\hline (T6) T5 + imersão em água 24 h & 75 & 68 & 12,8 & 33 \\
\hline
\end{tabular}

$\overline{* T e s t e ~ l o g-r a n k: ~} \chi^{2}=329,5$ gl e p = 0; - indica que não foi possível estimar o tempo mediano, pois menos de 50\% das sementes germinaram. Obs. = Observados; Esp. = Esperados.

difere significativamente dos demais em algum momento do tempo, quanto ao seu efeito na superação de dormência.

Observando-se as curvas de Kaplan-Meier (Figura 1), aceita-se o pressuposto deproporcionalidade de riscos ao longo do tempo em todos os tratamentos. A curva de sobrevivência de T6 está sempre abaixo da curva dos demais tratamentos, sugerindo que, para qualquer tempo de sobrevivência considerado, a probabilidade de não germinar é sempre menor para este tratamento em relação aos demais. Em todos os tratamentos a probabilidade de se observar sementes não germinadas diminui com o tempo, embora os tratamentos T1, T2 e T3 mantiveram probabilidade de não germinar sempre acima de $80 \%$.

A suposição de riscos proporcionais necessária para o modelo de Cox pode ser verificada de forma aproximada nas curvas de sobrevivência de Kaplan- 
Meier (Figura 1), cuja distância entre elas deve ser aproximadamente constante em todo o tempo. Para que a suposição seja válida, o gráfico dos resíduos padronizados de Schoenfeld, em função do tempo para cada um dos tratamentos (Figura 2), deve ser uma linha horizontal, pois a inclinação zero não indica evidências contra a proporcionalidade de riscos (COLOSIMO \& GIOLO 2006).

As estimativas dos coeficientes do modelo de Cox com as respectivas significâncias são apresentadas na Tabela 4. Os valores são interpretados como riscos relativos ou razão de riscos. Neste sentido, razões acima de 1 indicam sobrerrisco (maior capacidade de germinar) e valores entre 0 e 1 indicam proteção (menor capacidade de germinar), sempre em relação ao T1. Desta forma, as sementes submetidas ao tratamento com escarificação mecânica e imersão em água por 24 h (T6) têm 22,5 vezes maior capacidade de germinar (tempo para germinação mais curto) que sementes não submetidas à superação de dormência (T1). A interpretação é análoga para os tratamentos T4 e T5 com coeficientes significativos. Já os tratamentos T2 e T3 não diferiram significativamente da testemunha, revelando ineficiência.

A resposta germinativa obtida com a ruptura mecânica do tegumento impermeável das sementes foi positiva (T5 e T6), fato também confirmado por FREITAS et al. (2013) com sementes de Jatobá.

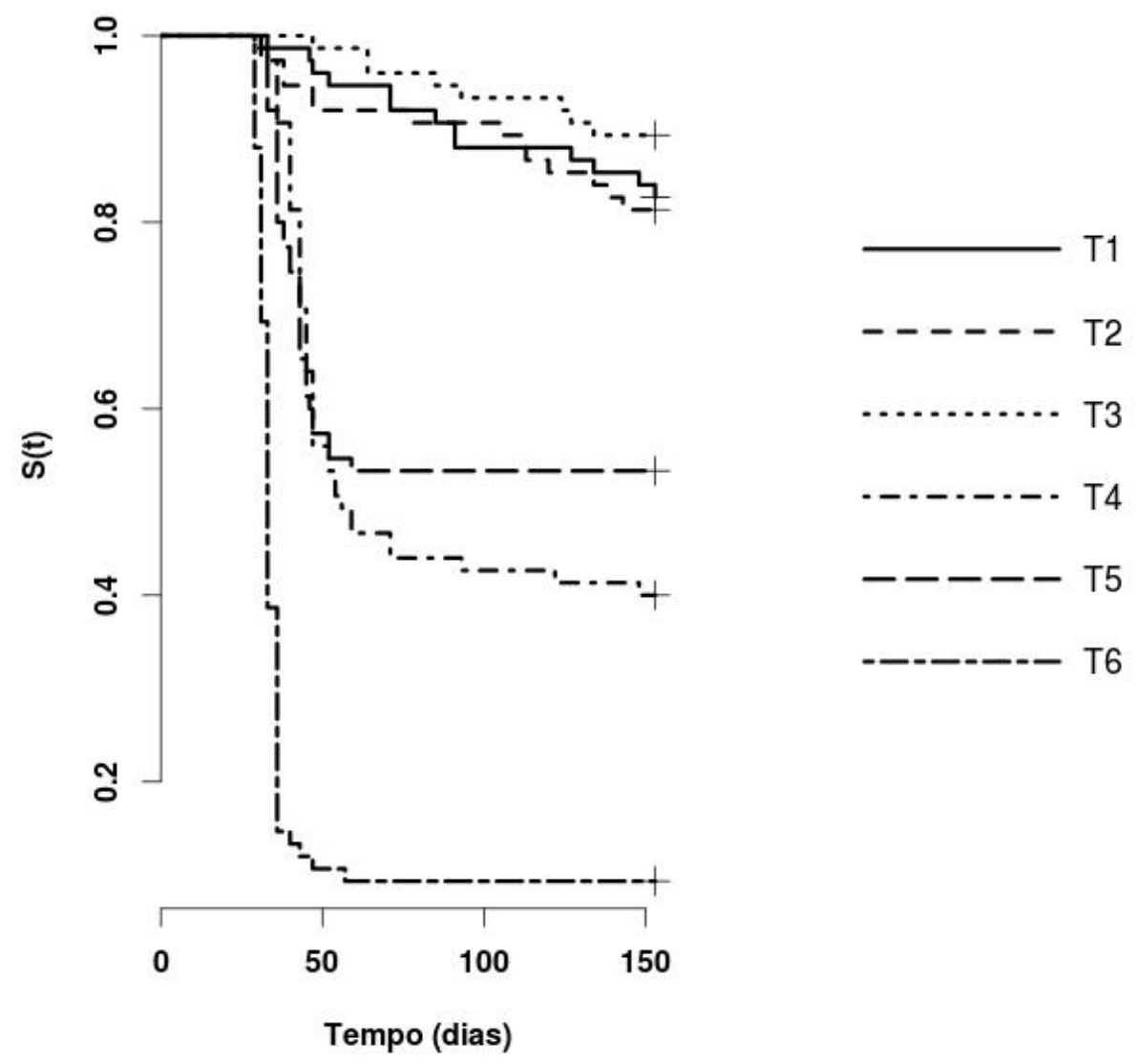

Figura 1 - Estimativas das curvas de Kaplan-Meier das funções de sobrevivência $(\mathrm{S}(\mathrm{t}))$ descrevendo o tempo para germinação de sementes de Jatobá (Hymenaea courbaril L.) em função do tratamento para a superação de dormência. T1 = testemunha; T2 e T3 = imersão em água à temperatura ambiente durante 120 h e $72 \mathrm{~h}$, respectivamente; T4 = imersão em água a $90^{\circ} \mathrm{C}$ e repouso nesta por $24 \mathrm{~h}$; T5 = escarificação mecânica; T6 = escarificação mecânica e imersão em água por $24 \mathrm{~h}$.

Figure 1 - Kaplan-Meier curves estimated of survival functions $(S(t))$ describing time to germination in Jatoba seeds (Hymenaea courbaril L) as a function of treatment to overcoming dormancy. $T 1=$ control; $T 2$ and $T 3=$ immersion in water at outdoor temperature for $72 \mathrm{~h}$ and $120 \mathrm{~h}$, respectively; $T 4=$ immersion in water at $90^{\circ} \mathrm{C}$ and this rest for $24 \mathrm{~h} ; \mathrm{T5}=$ mechanical scarification; T6 = mechanical scarification and soaking in water for $24 \mathrm{~h}$. 

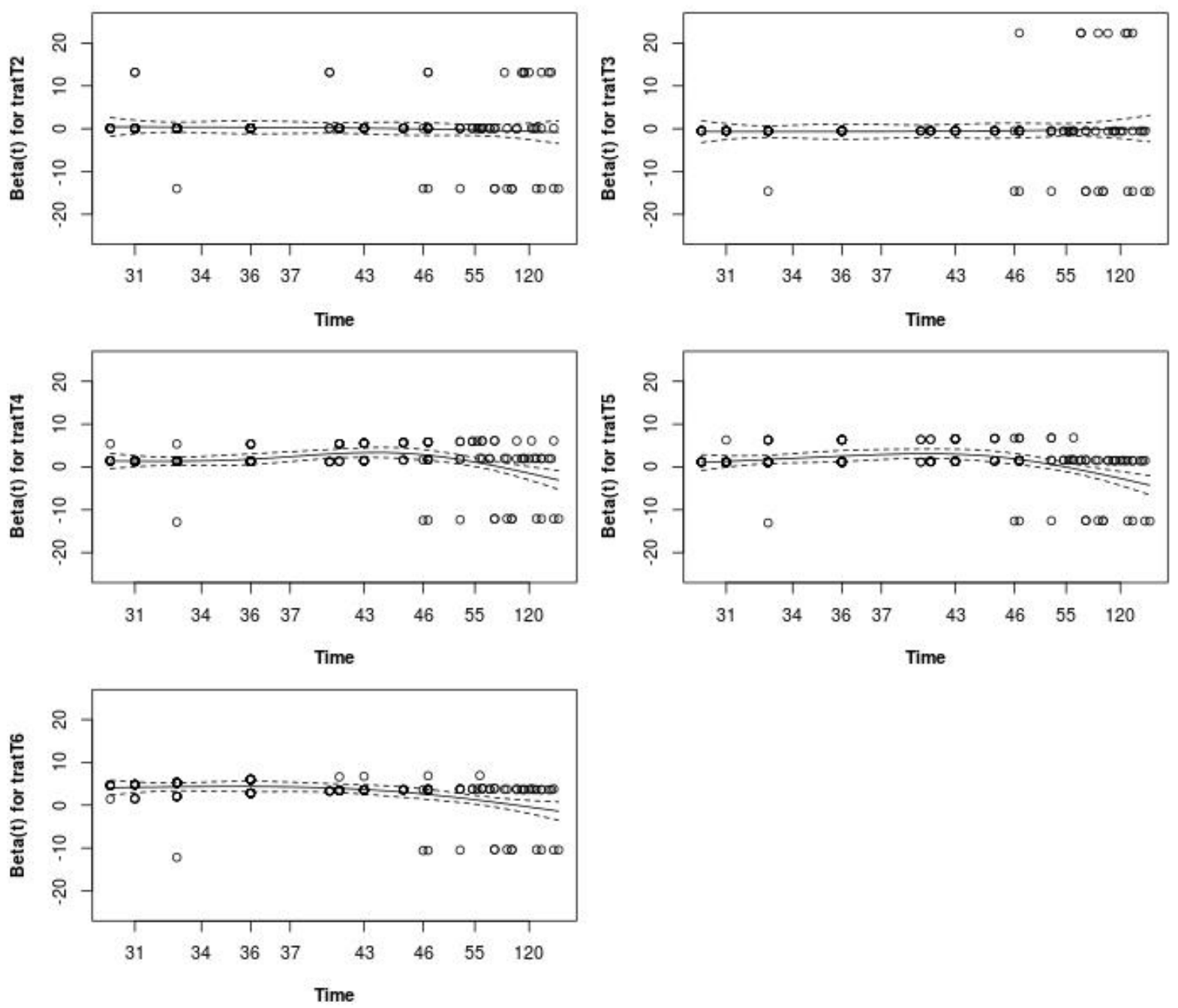

Figura 2 - Resíduos padronizados de Schoenfeld estimados para o modelo semiparamétrico de Cox em função dos tempos em cada tratamento para superação de dormência. T2 e T3 = imersão em água à temperatura ambiente durante $120 \mathrm{~h}$ e $72 \mathrm{~h}$, respectivamente; $\mathrm{T} 4=$ imersão em água a $90{ }^{\circ} \mathrm{C}$ e repouso nesta por 24 h; T5 = escarificação mecânica; T6 = escarificação mecânica e imersão em água por $24 \mathrm{~h}$.

Figure 2 - Schoenfeld standardized residuals estimated for the semiparametric Cox model as a function of time for each treatment to overcoming dormancy. T2 and T3 = immersion in water at outdoor temperature for $72 \mathrm{~h}$ and $120 \mathrm{~h}$, respectively; T4 = immersion in water at $90{ }^{\circ} \mathrm{C}$ and this rest for $24 \mathrm{~h}$; $\mathrm{T5}=$ mechanical scarification ; T6 = mechanical scarification and soaking in water for $24 \mathrm{~h}$.

Ao romper o tegumento, permite-se trocas gasosas e, em contato com a água, a absorção é facilitada, acelerando a germinação. Estudando dormência de sementes, AZEREDO et al. (2003) verificaram que lixar as sementes de $H$. courbaril e na sequência embebê-las em água à temperatura ambiente por $24 \mathrm{~h}$, foi o método mais eficiente para superar sua dormência. Da mesma forma, DE-CARVALHO et al. (2005) em sementes de Jatobá do cerrado (Hymenaea stigonocarpa), obtiveram o melhor resultado de germinação (53\%), em areia com serragem, após a escarificação mecânica das sementes com embebição em água destilada à temperatura ambiente por $24 \mathrm{~h}$.

O contato da semente com a água quente $\left(90{ }^{\circ} \mathrm{C}\right)$ seguido de repouso na mesma água (T4), aumenta a sua permeabilidade e facilita a germinação o que está em conformidade com FOWLER \& BIANCHETTI (2000), que consideram este procedimento eficiente para a superação da dormência em algumas espécies florestais. Porém BUSSATTO et al. (2013) obtiveram resultado insatisfatório de germinação com a imersão de sementes de $H$. 
Tabela 4 - Riscos relativos de germinação em cada tratamento para a superação de dormência estimados pelo modelo de Cox, em relação ao tratamento testemunha (T1).

Table 4 - Relative risks of germination in each treatment for overcoming dormancy estimated by the Cox model, compared to the control treatment (T1).

\begin{tabular}{lccc}
\hline Tratamento & exp (coef) & IC (95\%) & $\operatorname{Pr}(>|\mathrm{z}|)$ \\
\hline (T2) Imersão em água por $120 \mathrm{~h}$ & $1,10^{\mathrm{ns}}$ & $(0,52 ; 2,35)$ & 0,799 \\
(T3) Imersão em água por 72 h & $0,60^{\mathrm{ns}}$ & $(0,25 ; 1,45)$ & 0,257 \\
(T4) Imersão em água a $90^{\circ} \mathrm{C}$ & $4,70 *$ & $(2,53 ; 8,73)$ & $9,36.10^{-7}$ \\
(T5) Escarificação (2 mm) & $3,68^{*}$ & $(1,94 ; 6,95)$ & $6,20^{-5}$ \\
(T6) T5 + imersão em água 24 h & $22,50^{*}$ & $(12,29 ; 41,18)$ & $2,00^{-16}$
\end{tabular}

exp (coef): risco relativo ou razão de riscos - valores acima de 1 indicam sobrerrisco e valores entre 0 e 1 indicam proteção; IC (95\%): intervalo de confiança para a razão de riscos; "risco relativo difere siginificativamente do tratamento testemunha (T1). ${ }^{\text {ns }}$ não significativo.

courbaril em água a $90^{\circ} \mathrm{C}$ por dez minutos, atribuindo o insucesso à provável deterioração das sementes a essa temperatura.

Sementes tratadas unicamente com escarificação mecânica (T5, grosa 8"), apresentaram germinação abaixo de 50\% (46,67\%), similar ao observado por DE-CARVALHO et al. (2005) que obtiveram $37 \%$ de germinação em sementes de Jatobá do cerrado, em areia com serragem, somente escarificadas mecanicamente com esmeril elétrico. A germinação das sementes tratadas apenas com a imersão em água à temperatura ambiente por $120 \mathrm{~h}$ (T2) e 72 h (T3) não foram eficientes para a superação de dormência (Tabelas 2, 3 e 4).

\section{CONCLUSÕES}

Baseado nos resultados da análise de sobrevivência, os tratamentos diferem quanto ao efeito na superação de dormência de sementes de Jatobá, com destaque para o tratamento que combina escarificação de 2 mm e imersão em água a temperatura ambiente por $24 \mathrm{~h}$.

A técnica de análise de sobrevivência mostrouse adequada na comparação dos métodos de superação de dormência, com interpretação direta dos resultados e confiança nas estimativas.

\section{REFERÊNCIAS}

ALEXANDRE RS et al. 2009. Tratamentos físicos e químicos na superação de dormência em sementes de Enterolobium contortisiliquum (Vell.) Morong. Rev. Bras. Ciênc. Agrár. 4: 156-159.

ALMEIDA MB et al. 2011. Descrição morfológica do fruto e semente do Jatobá (Hymenaea courbaril L.). Revista Semiárido De Visu. 1: 107-115.

ANDRADE LA et al. 2010. Aspectos biométricos de frutos e sementes, grau de umidade e superação de dormência de Jatobá. Acta Scientiarum. 32: 293-299.

AZEREDO GA et al. 2003. Germinação de sementes de espécies florestais da Mata Atlântica (Leguminosae) sob condições de casa de vegetação. Pesqui. Agropecu. Trop. 33: 11-16.

BUSATTO PC et al. 2013. Superação de dormência em sementes de Jatobá (Hymenaea courbaril L.). Rev. Verde Agroecologia Desenvolv. Sustent. 8: 154-160.

CARVALHO MS et al. 2011. Análise de sobrevivência: teoria e aplicações em saúde. Rio de Janeiro, FIOCRUZ, 2.ed. 432p.

CARVALHO LR et al. 2006. Classificação de sementes florestais quanto ao comportamento no armazenamento. Rev. Bras. Sementes. 28: 15-25.

CARVALHO FILHO JLS et al. 2003. Produção de mudas de Jatobá (Hymenaea courbaril L.) em diferentes ambientes, recipientes e composições de substratos. CERNE. 9: 109118.

COLOSIMO EA \& GIOLO SR. 2006. Análise de sobrevivência aplicada. São Paulo: Edgard Blücher. 392p.

DE-CARVALHO PS et al. 2005. Germinação e dados biométricos de Hymenaea stigonocarpa Mart. Ex Hayne (Leguminosae Caesalpinoideae) - Jatobá do cerrado. Rev. anh. 6: 101-116.

FINCH-SAVAGE WE \& LEUBNER-METZGER, G. 2006. Seed dormancy and the control of germination. New 
Phytologist. 171: 501-523.

FOWLER JAP \& BIANCHETTI A. 2000. Dormência em sementes florestais. Documentos, 40. Colombo: Embrapa Florestas. 27p.

FREITAS AR et al. 2013. Superação de dormência de sementes de Jatobá. Pesqui. Florest. Bras. 33: 85-90. LEMOS FILHO JP et al. 1997. Germinação de sementes de Senna macranthera, Senna multijuga e Stryphnodendron polyphyllum. Pesqui. Agropecu. Bras. 32: 357-361.

R DEVELOPMENT CORE TEAM. 2014. R: A Language and Environment for Statistical Computing. Vienna, Austria: $\mathrm{R}$ Foundation for Statistical Computing. Disponível em: <http://www. R-project.org >. Acesso em: 22 abr. 2014.

THERNEAU TM. 2014. A Package for Survival Analysis in S. R package version 2.36-12. <URL: http://CRAN.Rproject.org/package=survival $>$. Acesso em: 22 abr. 2014. 